\title{
Identification of potential biomarkers in cervical cancer with combined public mRNA and miRNA expression microarray data analysis
}

\author{
SIZHE WANG and XIAOJIN CHEN \\ Department of Women Health Care, Beijing Chaoyang District Maternal and Child Health Care Hospital, \\ Beijing 100000, P.R. China
}

Received September 25, 2017; Accepted July 23, 2018

DOI: $10.3892 / \mathrm{ol} .2018 .9323$

\begin{abstract}
Cervical cancer is the fourth most prevalent malignancy in females worldwide. Early diagnosis is key to improving survival rates. Molecular biomarkers are an important method for diagnosing a number of types of cancer, including cervical cancer. The present study utilized public data from three mRNA microarray datasets and one microRNA dataset to analyze the key genes involved in cervical cancer. The mRNA and microRNA expression profile datasets (GSE9750, GSE46857, GSE67522 and GSE30656) were downloaded from the Gene Expression Omnibus database (GEO). Differentially expressed genes (DEGs) and microRNAs (DEMs) were screened using the online tool GEO2R. By using the DEGs consistent across the three mRNA datasets, a functional and pathway enrichment analysis was performed using the Database for Annotation, Visualization and Integrated Discovery. A protein-protein interaction (PPI) network was constructed and module analysis performed using the Search Tool for the Retrieval of Interacting Genes. Validated target genes of the DEMs were identified using the miRecords website. Using the identified target genes of the DEMs, a survival analysis was performed using the OncoLnc online tool. A total of 73 DEGs and 19 DEMs were screened from the microarray expression profile datasets. 'Integrin-mediated', 'proteolysis' and 'phosphoinositide 3 kinase-protein kinase 3' signaling pathways were the most enriched in the DEGs. Three of the DEGs, including Ras homolog family member B (RhoB), stathmin 1 (STMN1) and cyclin D1 (CCNB1) were validated DEM target genes. The OncoLnc survival analysis identified that RhoB was associated with a significantly longer overall survival, whereas STMN1 was associated with a significantly
\end{abstract}

Correspondence to: Dr Xiaojin Chen, Department of Women Health Care, Beijing Chaoyang District Maternal and Child Health Care Hospital, 25 East Third Ring Road of Beijing, Beijing 100000, P.R. China

E-mail: cyxxk2012@126.com

Key words: cervical cancer, biomarker, microarray, survival analysis reduced overall survival time in patients with cervical cancer. Finally, data from The Cancer Genome Atlas revealed an association between the mRNA expression levels of RhoB and STMN1, and the overall survival time for patients with cervical cancer. In conclusion, RhoB and STMN1 were identified as key genes that may provide potential targets for cervical cancer diagnosis and treatment.

\section{Introduction}

Cervical cancer is the fourth most prevalent type of malignancy in females worldwide (1). It is responsible for an estimated 520,000 new diagnoses and 270,000 mortalities annually (2). In China, it ranks as the eighth most frequent type of cancer in females, and the second most frequent cancer in females aged between 15 and 44. A previous study reported that $43 \%$ of diagnosed patients are $<45$, and $20-28 \%$ are $<40$ years old (3). In recent years, there has been a decrease in the incidence and mortality rates for cervical cancer in developed countries, which has been attributed to the effectiveness of screening tests. However, the incidence rate remains high in developing countries, accounting for $85 \%$ of all cases (4). Surgery is the curative treatment for cervical cancer. The earlier in disease progression a diagnosis is achieved, the better the prognosis and overall patient survival time are likely to be.

Sophisticated online tools combined with high-throughput analysis and general data availability enable the scientific community to uncover novel information regarding genes associated with cancer development at an unprecedented rate (5). Molecular biomarkers are associated with the detection/diagnosis of the disease, whereas prognostic biomarkers offer information about its course and the likelihood of recurrence (6). Predictive biomarkers estimate the response to treatment. The presence, absence or change in specific cell biomarkers may indicate the development of cancer. Ultimately, the identification and detection of these cancer-specific biomarkers may be of use in the early diagnosis and monitoring of the disease (7-9).

Microarrays, including DNA, microRNA, protein and antibody microarrays, are multiplex labs-on-a-chip. They can be used to assay significant amounts of biological material using high-throughput screening. This screening uses 
miniaturized and multiplexed parallel processing and detection methods. It is popular technology in recent years $(10,11)$ and is commonly used to obtain data regarding genetic alterations during tumorigenesis $(12,13)$. This data may be critical in the identification of biomarkers. In the present study, data from three mRNA microarray datasets and a microRNA dataset were used to analyze the differentially expressed genes (DEGs) and microRNAs (DEMs) in cervical cancer compared with normal cervical tissue. Bioinformatics methods, including function and pathway enrichment analysis using Kyoto Encyclopedia of Genes and Genomes (KEGG) and Gene Ontology (GO), and survival analysis using the OncoLnc tool, were used to identify the key genes in cervical cancer.

\section{Materials and methods}

Microarray datasets and data processing. A total of 3 human cervical cancer mRNA expression datasets [GSE9750 (14), GSE46857 (15) and GSE67522 (16)] and 1 human cervical cancer miRNA expression dataset [GSE30656 (17)] were selected randomly and downloaded from the Gene Expression Omnibus database (GEO). All the datasets contained a comparison between cervical cancer and normal cervix tissue samples. The three gene expression datasets included 54 cervical cancer tissue samples, and 38 normal cervix tissue samples from women without cervical cancer. The miRNA dataset comprised of 10 normal cervix tissue samples, and 19 squamous cell carcinoma or adenocarcinoma tissue samples. All four datasets were produced and uploaded to GEO by independent research groups. These datasets were based on 4 different Affymetrix platforms, including GPL96, GPL16690, GPL10558 and GPL6955 (Thermo Fisher Scientific, Inc., Waltham, MA, USA); these are all preferred platforms for biomarker cancer research.

GEO2R, an online tool comparing groups in GEO datasets, was used to identify DEGs and DEMs between cervical cancer and normal tissue samples. Genes were selected with the criteria of Benjamini and Hochberg adjusted $\mathrm{P}<0.01$, and $\log$ fold-change $>1$ or $<-1$. Finally, the 'Calculate and draw custom Venn diagrams' online tool (http://bioinformatics. psb.ugent.be/webtools/Venn/) was used to identify the significantly upregulated or downregulated genes across all three GEO mRNA datasets.

Protein-protein interaction (PPI) network construction and functional pathway enrichment analysis. The Search Tool for the Retrieval of Interacting Genes (STRING) database was used to identify and visualize the functional interactions between proteins (18). The Database for Annotation, Visualization and Integrated Discovery (DAVID), an online program that aids in the understanding of the biological function of genes, was used to perform a functional enrichment analysis of the DEGs, including Gene Ontology (GO) and Kyoto Encyclopedia of Genes and Genomes (KEGG) pathway enrichment analyses (19). A false discovery rate threshold was set at $\mathrm{P}<0.05$.

Validation of miRNA targets. The miRecords tool was used to identify the validated target genes of the DEMs. The miRecords tool provides a comprehensive search for scientific papers regarding miRNAs and their target genes from the National Centre for Biotechnology Information (20). The 'Calculate and draw custom Venn diagrams' tool was used to identify the target genes of the identified DEMs within the list of identified DEGs.

Survival analysis. The OncoLnc tool (www.oncolnc.org) was used to conduct an overall survival analysis for patients with cervical cancer. OncoLnc is an online tool for interactively exploring the survival data of 8,647 patients from 21 cancer studies in The Cancer Genome Atlas (TCGA), along with mRNA and miRNA RNA-Seq expression data from TCGA. The tool allows the production of Kaplan-Meier plots stratified by gene expression levels. Log-rank P-values in survival analysis were recorded. 80th (upper) percentiles and 20th (lower) percentiles were considered as high and low groups. The OncoLnc tool was also used to conduct an overall survival analysis for other types of cancer, including breast, lung, colorectal and prostate cancer. To research further into the association of cervical cancer with the DEGs regulated by DEMs, including Ras homolog family member B (RhoB) and Stathmin 1 (STMN1), all cervical cancer data were downloaded from TCGA. Using this data, the association between RhoB/STMN1 expression and survival time for patients with cervical cancer was analyzed.

DEGs basic expression state in different organs. The Human Protein Atlas (HPA; www.proteinatlas.org), an open-access database of the genome-wide expression of genes in various organ types (21), was used to identify the basic expression level of DEGs in different human organs. In the present study, three datasets were used; the HPA dataset (RNA-seq data mean values for different individual samples from each tissue reported as mean transcripts per million), the Genotype-Tissue Expression (GTEx) project dataset (RNA-seq data reported as median reads per kilobase per million mapped reads) and the Functional Annotation of the Mammalian Genome (FANTOM5) dataset (obtained through cap analysis of gene expression, reported as tags per million).

Statistical analysis. Statistical analysis was performed with GraphPad Prism 7 (GraphPad Software, Inc., La Jolla, CA, USA) and Microsoft Excel 2017 (Microsoft Corporation, Redmond, WA, USA). Kaplan-Meier analysis and log-rank test were used in overall survival analysis (by OncoLnc) and pathway enrichment analysis (by DAVID). ANOVA and a Student-Newman-Keuls-q post hoc test were used for multiple group comparisons. $\mathrm{P}<0.05$ was considered to indicate a statistically significant difference.

\section{Results}

Identification of DEGs in microarray datasets and PPI network construction. A total of 2,496,1,425 and 1,184 DEGs were identified in cervical cancer tissue compared with normal tissue samples in the GSE9750, GSE46857 and GSE67522 datasets, respectively. The 73 genes with a consistent trend in three microarrays towards being either upregulated or downregulated were considered DEGs in all three datasets (Fig. 1; Table I). Specifically, 45 genes were consistently upregulated and 28 genes were consistently 
Table I. List of 84 genes, 11 of which exhibited an inconsistent trend, that were identified as upregulated or downregulated using the GSE9750, GSE46857 and GSE67522 microarray datasets.

A, Upregulated DEGs

IGF1, KRT1, AHNAK, FHL1, PRRX1, CYP3A5, ZFP36, CCND1, IGFBP5, PALMD, APOD, PIK3R1, PGM5, FOSB, GSTM5, DCN, BAIAP2, SYNGR1, HOPX, CXCL14, MPZL2, LAMA2, CDH13, CRYAB, MAL, EGR1, ARG1, DUSP1, TGM3, DPT, RGS5, SDPR, SORBS1, FOS, RHOB, CXCL12, JAM2, SPARCL1, AQP1, COL17A1, CAB39L, SLC5A1, GPX3, PTGS1, PTGIS

\section{B, Downregulated DEGs}

BRCA1, LAMP3, ASF1B, RYR1, SMC4, AURKA, CKS1B, PCNA, SPP1, TYMS, APOL1, PLOD2, STMN1, CKS2, LYN, AGRN, CCNB2, PRC1, TOP2A, CXCL9, WDHD1, RSAD2, HMMR, MCM6, IFNAR2, PLAUR, CENPF, BIRC3

$\mathrm{C}$, Genes with an inconsistent trend

PEG3, NTRK2, BNIP3, MYH11, SVEP1, TRIM13, UNC93A, ARMCX1, IL17RC, KRT7, SLC6A8

DEG, differentially expressed genes.

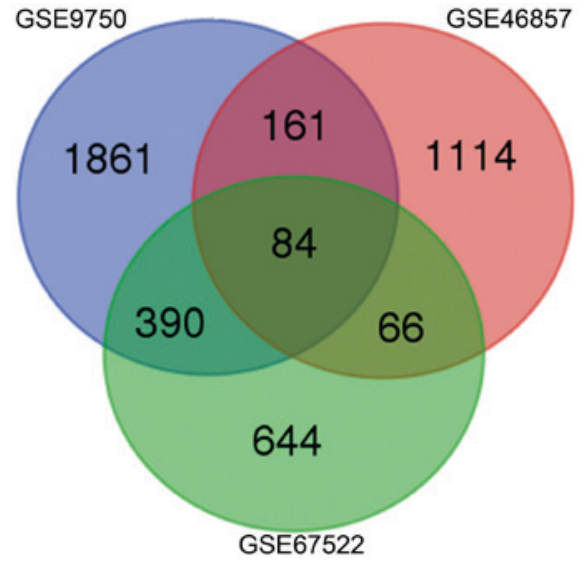

Figure 1. Identification of the differentially expressed genes in the GSE15471, GSE16515 and GSE28735 mRNA expression profile datasets.

downregulated. A PPI network was constructed of the DEGs (Fig. 2).

Functional pathway enrichment analysis. DAVID was used to analyze the potential biological functions and pathways of the identified DEGs. Biological processes including 'integrin-mediated signaling pathway', 'proteolysis' and 'collagen catabolic processes' were enriched in the DEGs. Furthermore, 7 KEGG pathways were enriched in the upregulated genes, including 'ECM-receptor interaction', 'focal adhesion' and 'PI3K-Akt signaling' pathways (Table II).

miRNA-DEG pairs. A total of 21 DEMs were identified in cervical cancer tissue compared with normal tissue in the GSE30656 dataset. Specifically, 19 miRNAs were upregulated and 2 were downregulated (Table III). By reference to miRecords, 246 validated gene targets for these miRNAs were identified. A PPI network was constructed for the targets (Fig. 3). It was identified that three of the validated DEM targets were DEGs (Fig. 4); cyclin D1 (CCND1) was among the validated gene targets of miR-1, while RhoB and STMN1 were among the predicted gene targets of miR-223.

Overall survival analysis. OncoLnc was used to analyze of the effect of CCND1, RhoB and STMN1 on the survival of patients with cervical cancer. It was identified that the high mRNA expression of RhoB was associated with a poor survival rate (log-rank $\mathrm{P}=0.0322$; Fig. $5 \mathrm{~A}$ ) whereas the low mRNA expression of STMN1 was associated with a poor survival rate (log-rank $\mathrm{P}=0.0046$; Fig. 5B). However, there was no significant association between CCND1 and overall survival time (Fig. 5C). An overall survival analysis was also conducted for the expression of these genes in other types of cancer, including breast, lung, colorectal and prostate cancer (Table IV). TCGA data from 263 patients with cervical cancer was divided into high (>50\% expression level) and low ( $<50 \%$ expression level) groups based on the expression of RhoB and STMN1. There were 64 patients in the RhoB high and STMN1 low group, which was referred to as group B. There were also 64 patients in the RhoB low and STMN1 high group, which was referred to as group D. The remaining patients were placed into either group A (RhoB high and STMN1 high) or group C (RhoB low and STMN1 low) (Fig. 6A and B). The patient survival time for group B was significantly decreased compared with group $\mathrm{D}(\mathrm{P}<0.05$; Fig. 6C). Additionally, the patient survival time for group B was the least out of the four groups, and it was significantly decreased compared with groups A, C and D $(\mathrm{P}<0.05$; Fig. 6C). Furthermore, the patient survival time for group $\mathrm{C}$ was the greatest out of the four groups, and it was significantly increased compared with groups $\mathrm{A}$ and $\mathrm{B}$ $(\mathrm{P}<0.05$; Fig. 6C). There was no significant difference between groups $\mathrm{C}$ and $\mathrm{D}$.

Basic expression state of DEGs in different organs. The Human Protein Atlas was used to assess the basic 
Table II. Functional/pathway enrichment analysis of the differentially expressed genes from 3 cervical cancer mRNA expression profiles.

\begin{tabular}{llll}
\hline Term & \multicolumn{1}{c}{ Description } & Count & P-value \\
\hline hsa05222 & Small cell lung cancer & 5 & $3.34 \times 10^{-3}$ \\
hsa04620 & Toll-like receptor signaling pathway & 5 & $6.45 \times 10^{-3}$ \\
hsa04510 & Focal adhesion & 6 & $1.59 \times 10^{-2}$ \\
hsa04512 & ECM-receptor interaction & 4 & $2.42 \times 10^{-2}$ \\
hsa05200 & Pathways in cancer & 7 & $3.29 \times 10^{-2}$ \\
hsa04062 & Chemokine signaling pathway & 5 & $4.94 \times 10^{-2}$ \\
hsa04150 & mTOR signaling pathway & 3 & $5.71 \times 10^{-2}$ \\
hsa00590 & Arachidonic acid metabolism & 3 & $6.51 \times 10^{-2}$ \\
hsa04110 & Cell cycle & 4 & $6.56 \times 10^{-2}$ \\
hsa05214 & Glioma & 3 & $7.99 \times 10^{-2}$ \\
hsa04115 & p53 signaling pathway & 3 & $9.11 \times 10^{-2}$ \\
hsa04730 & Long-term depression & 3 & $9.34 \times 10^{-2}$ \\
hsa05218 & Melanoma & 3 & $9.80 \times 10^{-2}$ \\
\hline
\end{tabular}

Table III. Differentially expressed miRNAs in cervical cancer screened out from miRNA expression microarray GSE30656 and their target genes which have been reported and validated using miRecords.

\begin{tabular}{|c|c|c|c|}
\hline miRNA & $\begin{array}{l}\text { Adjusted } \\
\text { P-value }\end{array}$ & $\begin{array}{l}\log \\
\text { fold change }\end{array}$ & Validated target genes \\
\hline miR-106b & $2.05 \times 10^{-7}$ & 1.02 & E2F1, CDKN1A, VEGFA, E2F1, CDKN1A, ITCH \\
\hline $\operatorname{miR}-125 b$ & $4.82 \times 10^{-4}$ & -1.44 & ERBB2, ERBB3, LIN28, BAK1, NTRK3, C10orf104, H3F3B, ADAMTS1, PERP \\
\hline miR-149 & $1.04 \times 10^{-4}$ & -1.14 & $\mathrm{~N} / \mathrm{A}$ \\
\hline $\operatorname{miR}-15 b$ & $1.42 \times 10^{-3}$ & 1.01 & BCL2, CCNE1, RECK, MKK4, RECK, BMI1 \\
\hline miR-16 & $5.47 \times 10^{-4}$ & 1.06 & TPPP3, BCL2, VEGFA, CCND1, PDCD4, RAB21, CADM1, SKAP2, WT1, BCL2 \\
\hline miR-192 & $3.98 \times 10^{-2}$ & 1.18 & DHFR, WNK1, RB1 \\
\hline $\operatorname{miR}-193 b$ & $2.69 \times 10^{-4}$ & -1.29 & PLAU, ESR1 \\
\hline miR-194 & $2.24 \times 10^{-2}$ & 1.07 & $\mathrm{~N} / \mathrm{A}$ \\
\hline miR-200a & $9.84 \times 10^{-3}$ & 1.07 & ZEB2, ZEB1 FOG2, ERBB2IP, BAP1, KLHL20 \\
\hline miR-203 & $1.57 \times 10^{-5}$ & -3.00 & N/A \\
\hline miR-205 & $2.16 \times 10^{-2}$ & -2.26 & ZEB2, ZEB1, VEGFA, INPPL1, ERBB3, PRKCE, MED1 \\
\hline miR-21 & $1.69 \times 10^{-5}$ & 2.10 & TPM1, NFIB, PDCD4, SERPINB5, CDKN1A, FAS, FAM3C, HIPK3, PRRG4, ACTA2 \\
\hline miR-223 & $1.38 \times 10^{-2}$ & 1.04 & NFIA, LMO2, LMO2, STMN1, RHOB, IRS1, FBXW7, EPB41L3 \\
\hline $\operatorname{miR}-370$ & $1.04 \times 10^{-3}$ & -2.37 & MAP3K8 \\
\hline miR-494 & $2.18 \times 10^{-2}$ & -1.22 & PTEN \\
\hline $\operatorname{miR}-565$ & $1.61 \times 10^{-2}$ & -1.04 & $\mathrm{~N} / \mathrm{A}$ \\
\hline miR-572 & $1.23 \times 10^{-2}$ & -1.04 & $\mathrm{~N} / \mathrm{A}$ \\
\hline $\operatorname{miR}-575$ & $1.02 \times 10^{-3}$ & -1.46 & $\mathrm{~N} / \mathrm{A}$ \\
\hline $\operatorname{miR}-630$ & $1.34 \times 10^{-2}$ & -1.20 & N/A \\
\hline miR-638 & $1.21 \times 10^{-2}$ & -1.37 & $\mathrm{~N} / \mathrm{A}$ \\
\hline miR-99a & $6.46 \times 10^{-4}$ & -1.25 & RAVER2, mTOR, IGF-IR, RPTOR, FGFR3 \\
\hline
\end{tabular}

N/A, not applicable.

expression level of RhoB and STMN1 in different organs. The results revealed that the expression level of RhoB in the cervix was relatively high compared with $>30$ human organ sample types. The expression of STMN1 in the cervix was low relative to the other sample types. However, the expression of RhoB and STMN1 in the cervix was not particularly high or low in comparison with normal organ samples (Fig. 7). 


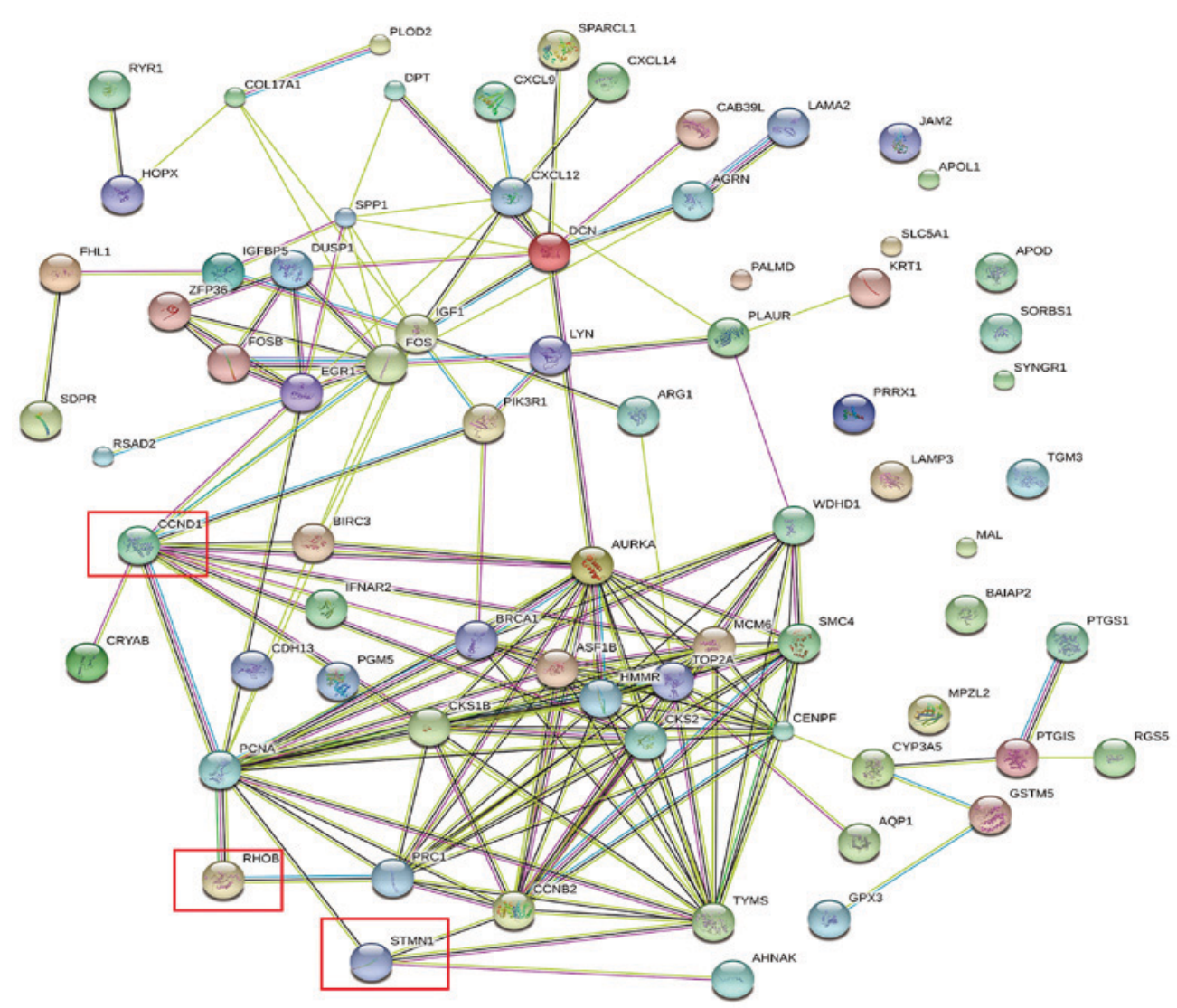

Figure 2. Protein-protein interaction network of all differentially expressed genes from the three mRNA expression profile datasets. The red boxes indicate potentially positive differentially expressed genes. CCND1, cyclin D1; STMN1, stathmin 1; RhoB, Ras homolog family member B.

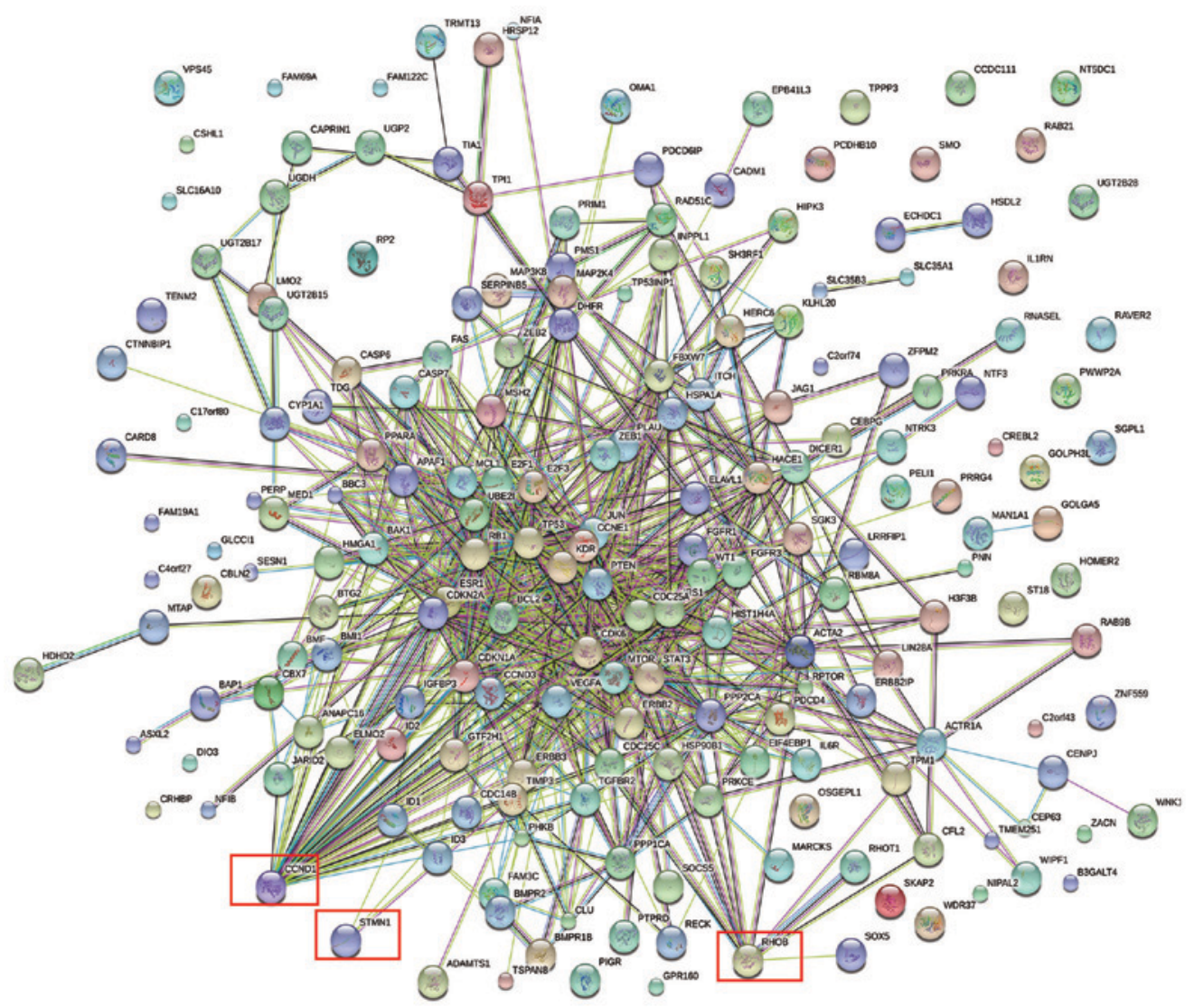

Figure 3. Protein-protein interaction network of differentially expressed genes based on one miRNA expression profiling dataset validated target genes. The red boxes indicate potentially positive differentially expressed genes. CCND1, cyclin D1; STMN1, stathmin 1; RhoB, Ras homolog family member B. 
Table IV. The prognostic value of two differentially expressed genes identified in patients with other types of cancer.

\begin{tabular}{lcc}
\hline Cancer type & Ras homolog family member B & Stathmin 1 \\
\hline Breast & 0.158 & 0.569 \\
Gastric & 0.046 & 0.431 \\
Lung & 0.339 & 0.127 \\
Ovarian & 0.062 & 0.293 \\
\hline
\end{tabular}

\section{DEGs in three mRNA microarrays}

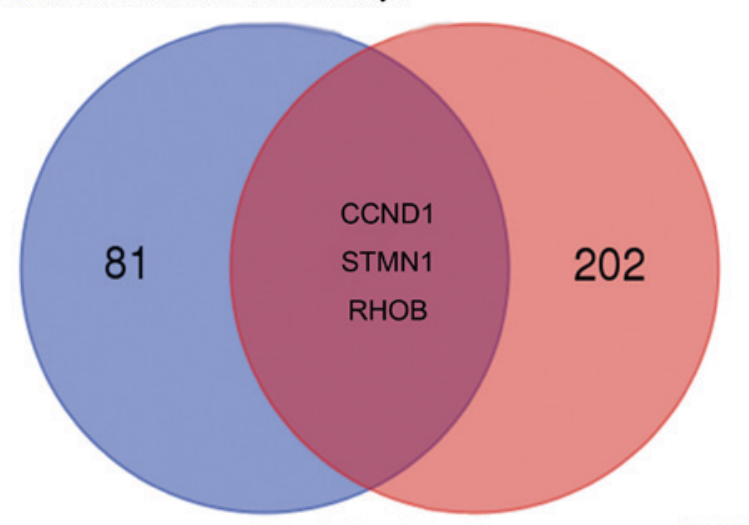

246 validated targets genes of DEMs

Figure 4. The comparison between differentially expressed genes based on three mRNA expression profiling datasets and the validated targets of differentially expressed miRNAs based on one miRNA expression profiling dataset. CCND1, cyclin D1; STMN1, stathmin 1; RhoB, Ras homolog family member B.

\section{Discussion}

The majority of cervical cancer patients live in economically underdeveloped areas, where the mortality rate is higher (22). Although cervical screening programs based on cervical cytology and human papilloma virus testing effectively reduce the incidence of cervical cancer, it is common for women in these areas not to accept standardized screening. This is largely due to social, religious and psychological factors (23). Therefore, novel circulating biomarkers to monitor critical molecular events common in cervical cancer may improve the detection of malignant lesions in primary screening and triage settings.

In the present study, three mRNA and one miRNA expression profile datasets were used to identify potential biomarkers in cervical cancer. These four datasets were based on microarrays produced by the same company, with four different platforms. Firstly, a comprehensive analysis of the DEGs and DEMs was performed, including GO and KEGG pathway enrichment analysis and PPI network analysis. Secondly, the DEGs were screened from the previously validated targets of the DEMs. A total of 73 DEGs were common to all three mRNA datasets, and 18 DEMs were identified from the microRNA dataset. Compared with existing studies, this screening was larger, and more comprehensive and random, due to it be conducted with a greater number of samples from different areas and a greater number of expression profiles.
The verified target genes of the DEMs were then obtained by reference to existing studies using miRecords. By comparing the verified target genes of the DEMs and the DEGs identified from the mRNA microarray, three potential genes were identified that may serve a key function in cervical cancer. Finally, it was identified through survival analysis that the high mRNA expression of RhoB was associated with poor overall survival (log-rank $\mathrm{P}=0.0322$; Fig. 5A), while the low mRNA expression of STMN1 was associated with poor overall survival (log-rank $\mathrm{P}=0.0046$; Fig. 5B). CCND1 expression was not significantly associated with overall survival (Fig. 5C).

Further analysis was performed using the survival data from TCGA. Data from 263 patients was divided into high or low groups based on the expression of RhoB and STMN1. It was identified that patients with higher RhoB and lower STMN1 expression experienced a significantly shorter overall survival time than patients with lower RhoB and higher STMN1 expression ( $\mathrm{P}<0.05$; Fig. 6C).

RhoB is a member of the Rho GTP-binding protein family located at 2p24.1 (24). RhoB may be a tumor suppressor as its expression level is decreased in a number of tumor cell types (25). Its expression is more downregulated in increasingly aggressive tumors, and the loss of RhoB is associated with a decreased overall survival time in certain types of cancer (26-28). RhoB is not mutated in all types of cancer; however, its altered expression and activity may be critical in cancer progression and the response to therapy. For example, RhoB expression is predictive of an epidermal growth factor receptor-tyrosine kinase inhibitor (EGFR-TKI) response; a EGFR-TKI/Akt inhibitor combination provides a clinical advantage in preventing resistance to EGFR-TKI for RhoB-positive tumor patients (26). A number of studies have identified a loss of RhoB expression in head, neck, gastric, renal and lung cancer (29-31). Studies of RhoB gene knockout in mice demonstrated that the frequency of chemically induced neoplastic transformation increased, and that the overexpression of RhoB in human cell lines results in the inhibition of signal transduction pathways associated with oncogenesis and tumor survival. The function of RhoB in cervical cancer has yet to be reported.

STMN1 is located at 1p36.11 and is also known as oncoprotein 18 . It is a $19-\mathrm{kDa}$ cytosolic protein that destabilizes microtubules in a phosphorylation-dependent manner and has been reported to be abundantly expressed in various types of cancer cell (32-34). STMN1 is also a biomarker in certain types of neoplasm. It serves important functions in cell cycle progression, mitosis, signal transduction and cell migration (35-37). There is no study reporting directly on the effect of STMN1 in cervical cancer.

In the present study, it was identified that upregulated RhoB was significantly associated with poor overall survival time in patients with cervical cancer, and that downregulated STMN1 was also significantly associated with poor overall survival. By comparing survival time with TCGA data, it was revealed that patients with higher levels of RhoB and lower levels of STMN1 have significantly shorter survival times. This supports that high RhoB and low STMN1 are associated with a worse prognosis, and that RhoB and STMN1 may be suitable for use in diagnosis and prognosis as biomarkers of cervical cancer. Furthermore, the basic expression levels of RhoB and STMN1 were assessed 

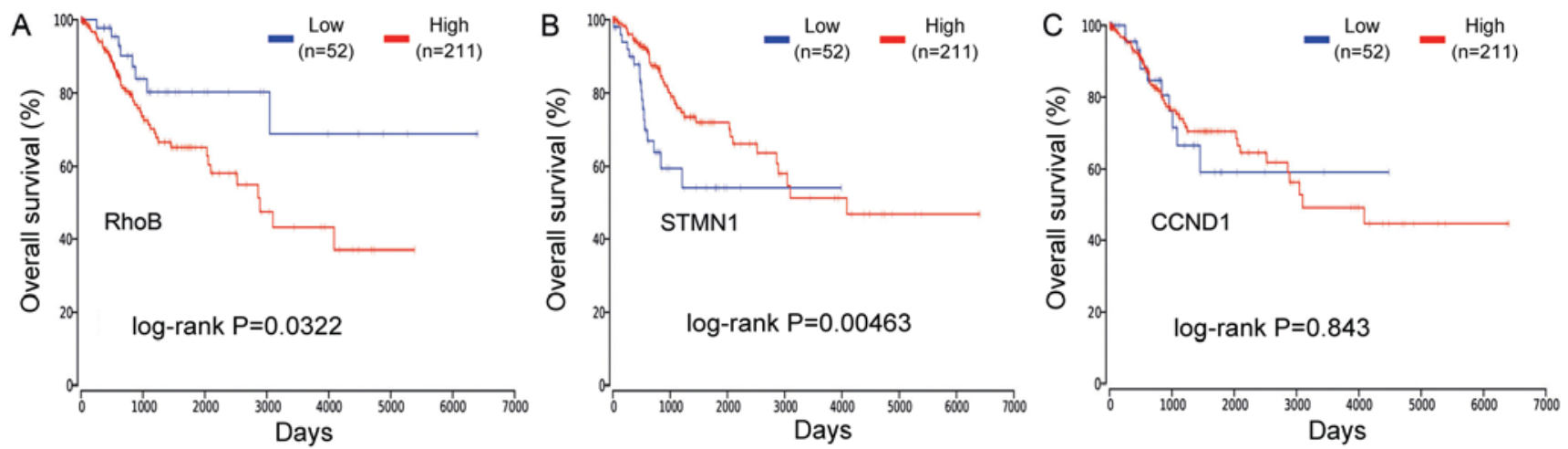

Figure 5. Analysis of the prognostic value of three differentially expressed genes in cervical cancer patients using The Cancer Genome Atlas data. (A) Prognostic value of RhoB, (B) STMN1, (C) CCND1. CCND1, cyclin D1; STMN1, stathmin 1; RhoB, Ras homolog family member B.

A Patients with higher RhoB

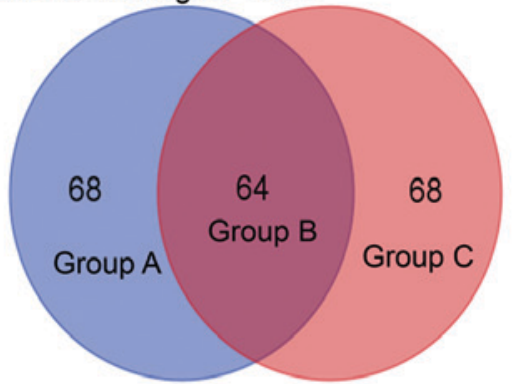

Patients with lower STMN1
B Patients with lower RhoB

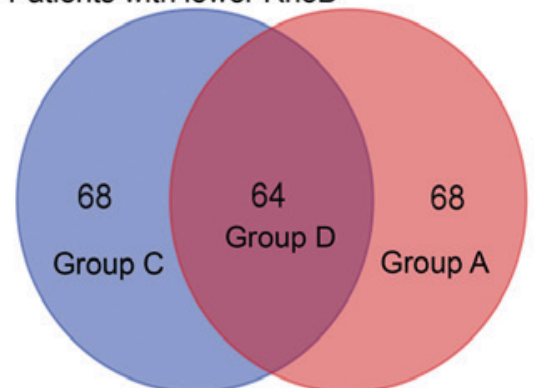

Patients with higher STM

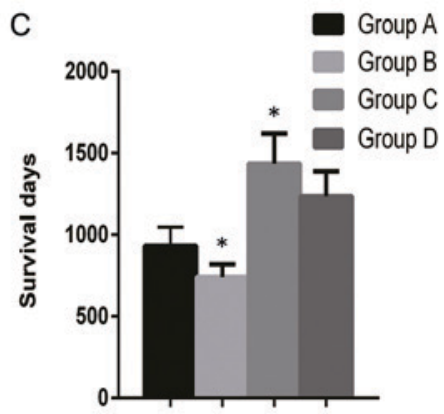

Figure 6. Comparison of survival times between the different expression level of differentially expressed genes. The grouping of cervical cancer patients with (A) high RhoB and low STMN1 and (B) low RhoB and high STMN1 using The Cancer Genome Atlas dataset. (C) Comparison between these groups with regard to survival time. STMN1, stathmin 1; RhoB, Ras homolog family member B.
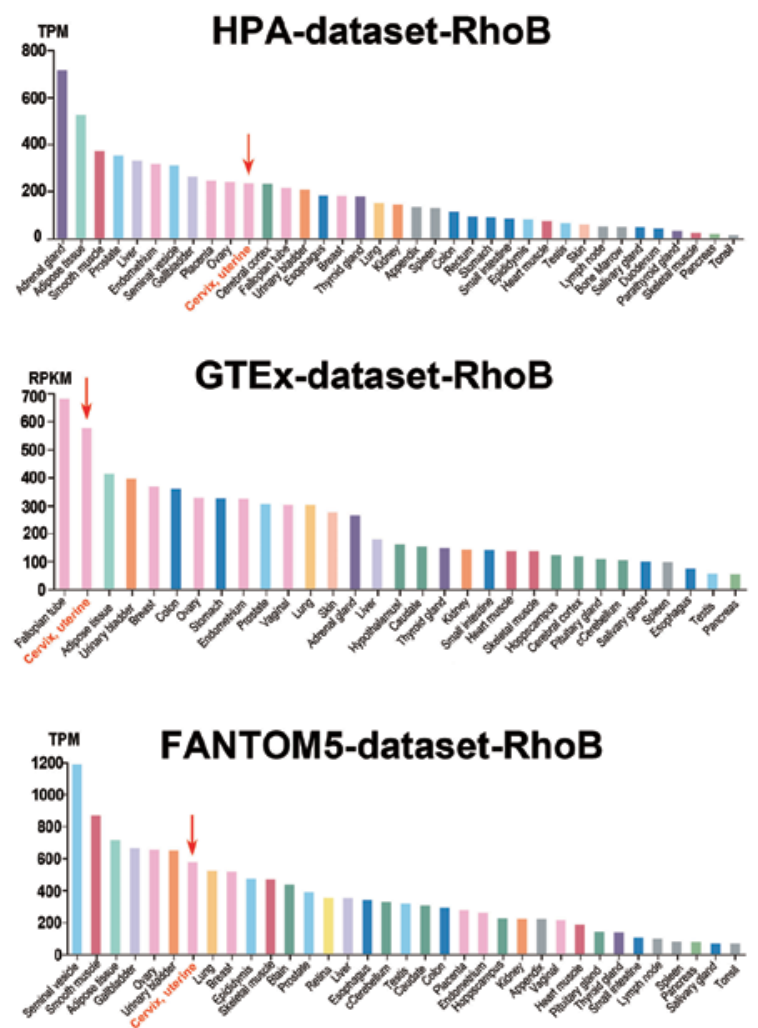
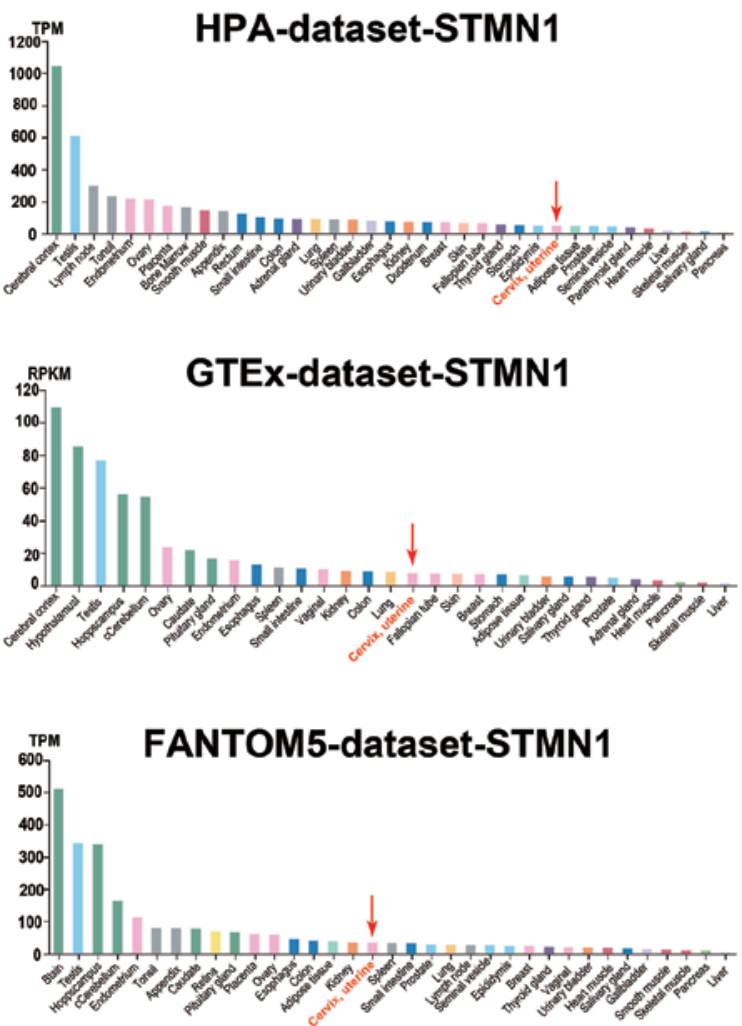

Figure 7. Basic expression state of differentially expressed genes in the cervix and other organs. STMN1, stathmin 1; RhoB, Ras homolog family member B; TPM, tags per million; RPKM, reads per kilobase of transcript per million mapped reads. 
in different tissue types using data mining methods. The results revealed that in normal cervical tissue samples, RhoB and STMN1 were not overexpressed or underexpressed in comparison with other sample types (Fig. 7). This indicates that it may be possible to perform a gain/loss of function analysis with normal cervix and cervical cancer cell lines in future studies. This would provide a more in-depth understanding of RhoB and STMN1 gene functions in cervical cancer.

In summary, mRNA and miRNA microarray expression datasets were screened for the identification of biomarkers in cervical cancer. In the present study, RhoB, STMN1 and the associated miR-223 may be critical in cervical cancer. We hypothesize that these genes and miRNA may serve as a key biomarker for predicting cervical cancer progression, and that the described method is used in clinical practice to improve the chance for an early diagnosis. For increased reliability and reproducibility, the method outlined in the present study will be repeated and further improved. Furthermore, we will study the functions and mechanism of the DEGs and DEMs identified in the present study. This will allow the greater understanding of broader clinical application prospects.

\section{Acknowledgements}

Not applicable.

\section{Funding}

No funding was received.

\section{Availability of data and materials}

The datasets generated and/or analyzed during the current study are available in the GEO repository, (https://www.ncbi. nlm.nih.gov/geo).

\section{Authors' contributions}

SW designed this research and collected the datasets. XC and SW analyzed the data and were involved in writing and revising the manuscript.

\section{Ethics approval and consent to participate}

Not applicable.

\section{Patient consent for publication}

Not applicable.

\section{Competing interests}

The authors declare that they have no competing interests.

\section{References}

1. Siegel RL, Miller KD and Jemal A: Cancer statistics, 2016. CA Cancer J Clin 66: 7-30, 2016

2. De AngelisR,Sant M,Coleman MP,FrancisciS,BailiP,PierannunzioD, Trama A, Visser O, Brenner H, Ardanaz E, et al: Cancer survival in Europe 1999-2007 by country and age: Results of EUROCARE-5-a population-based study. Lancet Oncol 15: 23-34, 2014.
3. Chen W, Zheng R, Baade PD, Zhang S, Zeng H, Bray F, Jemal A, Yu XQ and He J: Cancer statistics in China, 2015. CA Cancer J Clin 66: 115-132, 2016.

4. Vaccarella S, Laversanne M, Ferlay J and Bray F: Cervical cancer in Africa, Latin America and the Caribbean, and Asia: Regional inequalities and changing trends. Int J Cancer 141: 1997-2001, 2017.

5. Williams SP and McDermott U: The pursuit of therapeutic biomarkers with high-throughput cancer cell drug screens. Cell Chem Biol 24: 1066-1074, 2017.

6. Sun HH, Vaynblat A and Pass HI: Diagnosis and prognosis-review of biomarkers for mesothelioma. Ann Transl Med 5: 244, 2017.

7. Abdollah F, Dalela D, Haffner MC, Culig Z and Schalken J: The role of biomarkers and genetics in the diagnosis of prostate cancer. Eur Urol Focus 1: 99-108, 2015.

8. Patel SA and DeMichele A: Adding adjuvant systemic treatment after neoadjuvant therapy in breast cancer: Review of the data. Curr Oncol Rep 19: 56, 2017.

9. Verdaguer H, Saurí T and Macarulla T: Predictive and prognostic biomarkers in personalized gastrointestinal cancer treatment. J Gastrointest Oncol 8: 405-417, 2017.

10. Herrera-Marcos LV, Lou-Bonafonte JM, Arnal C, Navarro MA and Osada J: Transcriptomics and the mediterranean diet: A systematic review. Nutrients 9: E472, 2017.

11. Hong HJ, Koom WS and Koh WG: Cell microarray technologies for high-throughput cell-based biosensors. Sensors (Basel) 17: E1293, 2017.

12. Shimizu D, Kanda M and Kodera Y: Review of recent molecular landscape knowledge of gastric cancer. Histol Histopathol 33: $11-26,2018$

13. Syed P, Gidwani K, Kekki H, Leivo J, Pettersson K and Lamminmäki U: Role of lectin microarrays in cancer diagnosis. Proteomics 16: 1257-1265, 2016.

14. Scotto L, Narayan G, Nandula SV, Arias-Pulido H, Subramaniyam S, Schneider A, Kaufmann AM, Wright JD, Pothuri B, Mansukhani M and Murty VV: Identification of copy number gain and overexpressed genes on chromosome arm 20q by an integrative genomic approach in cervical cancer: Potential role in progression. Genes Chromosomes Cancer 47: 755-765, 2008.

15. Thomas A, Mahantshetty U, Kannan S, Deodhar K, Shrivastava SK, Kumar-Sinha C and Mulherkar R: Expression profiling of cervical cancers in Indian women at different stages to identify gene signatures during progression of the disease. Cancer Med 2: 836-848, 2013.

16. Sharma S, Mandal P, Sadhukhan T, Roy Chowdhury R, Ranjan Mondal N, Chakravarty B, Chatterjee T, Roy S and Sengupta S: Bridging links between long noncoding RNA HOTAIR and HPV oncoprotein E7 in cervical cancer pathogenesis. Sci Rep 5: 11724, 2015.

17. Wilting SM, Snijders PJ, Verlaat W, Jaspers A, van de Wiel MA, van Wieringen WN, Meijer GA, Kenter GG, Yi Y, le Sage C, et al: Altered microRNA expression associated with chromosomal changes contributes to cervical carcinogenesis. Oncogene 32: 106-116, 2013.

18. Szklarczyk D, Franceschini A, Wyder S, Forslund K, Heller D, Huerta-Cepas J, Simonovic M, Roth A, Santos A, Tsafou KP, et al: STRING v10: Protein-protein interaction networks, integrated over the tree of life. Nucleic Acids Res 43 (Database Issue): D447-D452, 2015.

19. Huang da W, Sherman BT and Lempicki RA: Systematic and integrative analysis of large gene lists using DAVID bioinformatics resources. Nat Protoc 4: 44-57, 2009.

20. Xiao F, Zuo Z, Cai G, Kang S, Gao X and Li T: miRecords: An integrated resource for microRNA-target interactions. Nucleic Acids Res 37 (Database Issue): D105-D110, 2009.

21. Uhlen M, Zhang C, Lee S, Sjöstedt E, Fagerberg L, Bidkhori G, Benfeitas R, Arif M, Liu Z, Edfors F, et al: A pathology atlas of the human cancer transcriptome. Science 357: eaan2507, 2017.

22. Aranda S, Berkley S, Cowal S, Dybul M, Evans T, Iversen K, Moeti M, Osotimehin B, Peterson S, Piot P, et al: Ending cervical cancer: A call to action. Int J Gynaecol Obstet 138 (Suppl 1): S4-S6, 2017.

23. Huchko MJ, Maloba M, Nakalembe M and Cohen CR: The time has come to make cervical cancer prevention an essential part of comprehensive sexual and reproductive health services for HIV-positive women in low-income countries. J Int AIDS Soc 18 (Suppl 5): 20282, 2015.

24. Thumkeo D, Watanabe S and Narumiya S: Physiological roles of Rho and Rho effectors in mammals. Eur J Cell Biol 92: 303-315, 2013. 
25. Schaefer A, Reinhard NR and Hordijk PL: Toward understanding RhoGTPase specificity: Structure, function and local activation. Small GTPases 5: 6, 2014.

26. Calvayrac O, Mazières J, Figarol S, Marty-Detraves C, Raymond-Letron I,BousquetE,Farella M,Clermont-TaranchonE, Milia J, Rouquette I, et al: The RAS-related GTPase RHOB confers resistance to EGFR-tyrosine kinase inhibitors in non-small-cell lung cancer via an AKT-dependent mechanism. EMBO Mol Med 9: 238-250, 2017.

27. Merkus D and Schermuly RT: Farnesylation of RhoB: The cancer hypothesis of pulmonary hypertension revisited. Cardiovasc Res 113: 249-250, 2017.

28. Vega FM and Ridley AJ: The RhoB small GTPase in physiology and disease. Small GTPases: 1-10, 2016.

29. Chen W, Niu S, Ma X, Zhang P, Gao Y, Fan Y, Pang H, Gong H, Shen D, Gu L, et al: RhoB acts as a tumor suppressor that inhibits malignancy of clear cell renal cell carcinoma. PloS One 11: e0157599, 2016.

30. Delmas A, Cherier J, Pohorecka M, Medale-Giamarchi C, Meyer N, Casanova A, Sordet O, Lamant L, Savina A, Pradines A and Favre G: The c-Jun/RHOB/AKT pathway confers resistance of BRAF-mutant melanoma cells to MAPK inhibitors. Oncotarget 6: 15250-15264, 2015.

31. Marlow LA, Bok I, Smallridge RC and Copland JA: RhoB upregulation leads to either apoptosis or cytostasis through differential target selection. Endocr Relat Cancer 22: 777-792, 2015.

32. Bai T, Yokobori T, Altan B, Ide M, Mochiki E, Yanai M, Kimura A, Kogure N, Yanoma T, Suzuki M, et al: High STMN1 level is associated with chemo-resistance and poor prognosis in gastric cancer patients. Br J Cancer 116: 1177-1185, 2017.
33. Dou YD, Zhao H, Huang T, Zhao SG, Liu XM, Yu XC, Ma ZX, Zhang YC, Liu T, Gao X, et al: STMN1 promotes progesterone production via StAR up-regulation in mouse granulosa cells. Sci Rep 6: 26691, 2016.

34. Zhang HQ, Guo X, Guo SQ, Wang Q, Chen XQ, Li XN and Guo LS: STMN1 in colon cancer: Expression and prognosis in Chinese patients. Eur Rev Med Pharmacol Sci 20: 2038-2044, 2016.

35. Chen Y, Zhang Q, Ding C, Zhang X, Qiu X and Zhang Z: Stathmin1 overexpression in hypopharyngeal squamous cell carcinoma: A new promoter in FaDu cell proliferation and migration. Int J Oncol 50: 31-40, 2017.

36. Li J, Hu GH, Kong FJ, Wu KM, He B, Song K and Sun WJ: Reduced STMN1 expression induced by RNA interference inhibits the bioactivity of pancreatic cancer cell line Panc-1. Neoplasma 61: 144-152, 2014

37. Li X, Wang L, Li T, You B, Shan Y, Shi S, Qian L and Cao X: STMN1 overexpression correlates with biological behavior in human cutaneous squamous cell carcinoma. Pathol Res Pract 211: 816-823, 2015 .

(i) (9) This work is licensed under a Creative Commons Attribution-NonCommercial-NoDerivatives 4.0 International (CC BY-NC-ND 4.0) License. 